\title{
MS03-P23 | The Crystal Structure Of The Major Olive Pollen Allergen Ole E 1
}

Wortmann, Judith (University of Graz, Graz, AUT); Hofer, Gerhard (University of Graz, Graz, AUT); Dorofeeva, Yulia (University of Graz, Graz, AUT); Focke-Tejkl, Margarete (University of Graz, Graz, AUT); Aschauer, Philipp (University of Graz, Graz, AUT); Pavkov-Keller, Tea (University of Graz, Graz, AUT); Valenta, Rudolf (University of Graz, Graz, AUT); Keller, Walter (University of Graz, Graz, AUT)

In the field of molecular allergology, structural investigations of important allergens are crucial for the development of hypoallergenic derivatives and the design of efficient vaccines. An important allergen source in the Mediterranean area is the olive tree pollen. Its main allergen is Ole e 1 which is recognized by $90 \%$ of atopic individuals with olive tree pollinosis. This work elucidates a new structural understanding of this protein family.

Recombinant Ole e 1 was produced natively folded in E. coli, characterized with biophysical methods and compared to natural Ole e 1 . The three-dimensional structure was solved using X-ray crystallography. Peptidebased IgE-binding and inhibition assays were performed to map the dominant IgE-epitope regions of Ole e 1.

We here present the 3D crystal structure of the major olive tree pollen allergen Ole e 1, the prototypic member of the Ole e 1-like family. The structure exhibits a 6-stranded $\beta$-barrel in the core, resembling the fold of Pla I 1, which is the only other known structure of this allergen family (PDB-Code: 4Z8W). Additionally, the extended C-terminus, which is missing in the homologous structure, forms an $\alpha$-helix that serves as the interface for a homodimer formation. Stable dimer formation was also proven in solution. Immunological characterization revealed the high allergenicity of the allergen and enabled the determination of IgE-epitope regions. 